\title{
Primordial black hole production during preheating in a chaotic inflationary model
}

\author{
E. Torres-Lomas $1, *$ and L. Arturo Ureña-López ${ }^{1,0}$ \\ ${ }^{1}$ Departamento de Física, DCI, Campus León, CP 37150, \\ Universidad de Guanajuato, León, Guanajuato, México
}

\begin{abstract}
In this paper we review the production of primordial black holes (PBHs) during preheating after a chaotic inflationary model. All relevant equations of motion are solved numerically in a modified version of HLattice, and we then calculate the mass variance to determine structure formation during preheating. It is found that production of $\mathrm{PBHs}$ can be a generic result of the model, even though the results seem to be sensitive to the values of the smoothing scale. We consider a constraint for overproduction of PBHs that could uncover some stress between inflation-preheating models and observations.
\end{abstract}

PACS numbers: 95.30Cq, 95.30.Tg, 98.80.Cq,97.60.Lf

Keywords: Scalar fields, preheating, primordial black holes.

\section{INTRODUCTION}

Recent research about the implications of a preheating stage on observable cosmological parameters (CMB, microhalos of dark matter, non-gaussianity) have been reported. In particular PBHs formation has been analysed in different models, it was found that an overproduction of these objects may appear but no conclusive results have been reported [1-3].

The primordial black holes (PBHs) production during inflation can play an important role in the physics after inflation, for example, by producing the reheating of the universe [4]. This PBHs production may appear only in some inflationary theories but not in a generic model. Despite of this, $\mathrm{PBHs}$ formation can occur during a preheating phase at the graceful exit from inflation [5-10]. The PBHs production that appears due to the explosive excitement of matter scalar perturbations has been widely explored both analytically and numerically, in cases in which metric perturbations are not taken into account. On the other hand, the production of the metric perturbations also has been studied but for different reasons, but with minor progress in the numerical part.

The complete analysis of the dynamics of a Universe during the preheating stage, with the consideration of both the redispersion and the feedback with the perturbations of the metric, can only be addressed by numerically solving the full Einstein's equations. In this paper we show the results of numerical evolution of the mass variance as a measure of structure formation in a model with two scalar fields, an inflaton scalar field $\phi$ and a spectator $\chi$, where the potential for chaotic inflation $\sim m^{2} \phi^{2}$ and an interaction term $\sim \phi^{2} \chi^{2}$ have been considered. The numerical solution is calculated by HLattice code [11], which realizes a full 3dimensional integration of the equations of motion. In this respect, our results do not rely on the assumption that matter and metric perturbations evolve separately.

A description of the paper is as follows. First of all, we provide the mathematical basis for the general study of preheating models. Throughout the numerical simulations, matter and metric perturbations are calculated, and the particle production associated with each scalar field at the end of the simulation is also reported. The initial and final spectrum of the curvature perturbation and the evolution of the mass variance are calculated as a measure of structure formation, with special interest in the conditions under which we may have PBHs overproduction: $\sigma \geq \sigma_{*}$. Finally, we discuss the cosmological implications that may arise from such an overproduction of PBHs. 


\section{PREHEATING}

We consider a preheating model where the matter Lagrangian $\mathcal{L}_{\text {mat }}$, that includes an inflaton field $\phi$, and a spectator field $\chi$, is given by ${ }^{1}$

$$
\mathcal{L}_{m a t}=\frac{1}{2} \phi_{, \alpha} \phi^{, \alpha}+\frac{1}{2} \chi, \alpha \chi^{, \alpha}+\frac{1}{2} m^{2} \phi^{2}+\frac{1}{2} g^{2} \phi^{2} \chi^{2}
$$

where $m=10^{-6} M_{P l}$, which is an typical mass value for the chaotic inflationary model [12], and $g^{2}$ is the coupling constant between the scalar fields. We also assume a flat background Friedmann-Robertson-Walker (FRW) geometry with metric perturbations given in the synchronous gauge as [13]

$$
d s^{2}=a^{2}(\tau)\left(-d \tau^{2}+\left(\delta_{i j}+h_{i j}\right) d x^{i} d x^{j}\right)
$$

where the metric perturbation $h_{i j}$ can be decomposed as

$$
h_{i j}=h \delta_{i j} / 3+h_{i j}^{\|}+h_{i j}^{\perp}+h_{i j}^{T},
$$

where $h \delta_{i j} / 3+h_{i j}^{\|}$represents the scalar part of the perturbation, whereas $h_{i j}^{\perp}$ and $h_{i j}^{T}$ represent the vectorial and tensorial parts, respectively.

In this work, our main concern are scalar perturbations, so it will be useful to define the parametrization of the scalar part $h_{i j}^{[S]} \equiv h \delta_{i j} / 3+h_{i j}^{\|}$in Fourier space as

$$
h_{i j}^{[S]}(\vec{x}, \tau)=\int d^{3} k e^{i \vec{k} \cdot \vec{x}}\left\{\hat{k}_{i} \hat{k}_{j} h(\vec{k}, \tau)+\left(\hat{k}_{i} \hat{k}_{j}-\frac{1}{3} \delta_{i j}\right) 6 \eta(\vec{k}, \tau)\right\}, \quad \vec{k}=k \hat{k}
$$

where the trace $h$ is used for both the real space and the Fourier space. It is convenient to define also the curvature perturbation in Fourier space, $\zeta_{k}$, as

$$
\zeta_{k} \equiv-\eta_{k}+\frac{\delta \rho_{k}^{S}}{3(\langle\rho\rangle+\langle p\rangle)},
$$

which by definition is a gauge-invariant quantity [14, 15]. Here, $\langle\rho\rangle$ and $\langle p\rangle$ are the mean values of the energy density and the pressure, respectively, that are defined as

$$
\begin{aligned}
& \langle\rho\rangle=\left\langle\frac{1}{2} \dot{\phi}^{2}+\frac{1}{2} \dot{\chi}^{2}+\frac{1}{2 a^{2}}|\nabla \phi|^{2}+\frac{1}{2 a^{2}}|\nabla \chi|^{2}+\frac{1}{2} m^{2} \phi^{2}+\frac{1}{2} g^{2} \phi^{2} \chi^{2}\right\rangle, \\
& \langle p\rangle=\left\langle\frac{1}{2} \dot{\phi}^{2}+\frac{1}{2} \dot{\chi}^{2}-\frac{1}{6 a^{2}}|\nabla \phi|^{2}-\frac{1}{6 a^{2}}|\nabla \chi|^{2}-\frac{1}{2} m^{2} \phi^{2}-\frac{1}{2} g^{2} \phi^{2} \chi^{2}\right\rangle,
\end{aligned}
$$

where $\langle\cdot\rangle$ means the spatial averaging in the 3 dimensional lattice.

We have calculated numerically the time evolution of the curvature perturbation (see Eq. (5)) for a Universe in the epoch of preheating, using the free numerical code HLattice [11]. This code realizes a full integration of the Einstein-Klein-Gordon equations of motion for two scalar fields, within a $3+1$ set up in the synchronous gauge; a complete analysis of the numerical methods used and the accuracy of the numerical results can be found in[11]. The metric perturbations are evolved by solving the Einstein equations of this model, without any explicit separation in homogeneous and inhomogeneous variables.

The initial conditions are such that the inflaton is oscillating around the minimum of the potential. The initial fields are initialized with a random gaussian fluctuations according to values a Bunch-Davis vacuum [16]. The value of the mass and the coupling constant are $m=10^{-6} M_{p l}$ and $g^{2}=2.5 \times 10^{-7}$,respectively.

To exhibit the dynamics of the matter during preheating Fig. 1 (left) shows the final spectrum of occupation numbers $n_{k}^{\phi}$ and $n_{k}^{\chi}$ associated with the scalar fields $\phi$ and $\chi$, respectively. This growth of $n_{k}^{\chi}$ produced by parametric resonances has been previously reported in the literature [17, 18]. In Fig 1 (right) the power spectrum of curvature perturbation at the end of inflation $P\left(\zeta_{k}\right)_{\text {end }}$ and at the end of preheating $P\left(\zeta_{k}\right)_{r e h}$ are shown. The growth of $P\left(\zeta_{k}\right)$ is a product of the growth of both the matter and metric perturbations (See (5)). This growth of the curvature perturbation should produce the structure formation that could possibly evolve to form PBHs.

\footnotetext{
1 The name spectator comes from considering that during inflation the whole dynamic was determined solely by the inflaton field $\phi$. However, a more realistic treatment would also consider the effects of the spectator $\chi$ field during inflation.
} 

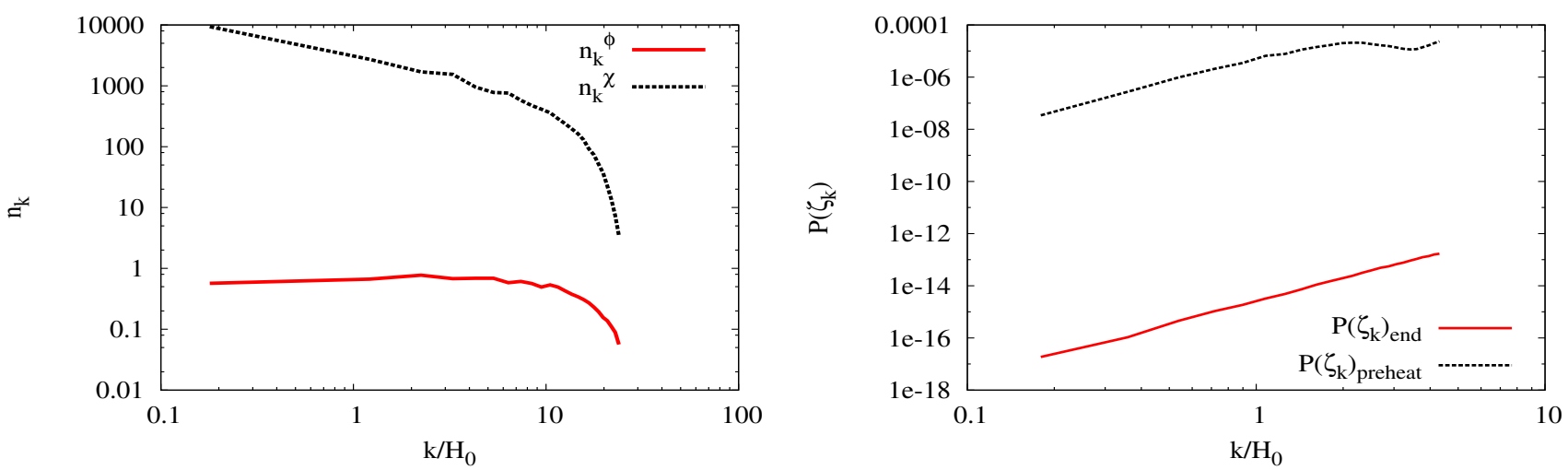

FIG. 1: (Left) Final spectrum of occupation numbers $n_{k}^{\phi}$ and $n_{k}^{\chi}$. This growth of $n_{k}^{\chi}$ is produced by parametric resonances. The production of light particles with a high kinetic energy can increase the temperature to produce a radiation dominated Universe. (Right) The power spectrum of curvature perturbation at the end of inflation $P\left(\zeta_{k}\right)_{\text {end }}$ and at the end of preheating $P\left(\zeta_{k}\right)_{r e h}$. This growth of the curvature perturbation should produce PBHs formation. See the text for more details.

\section{PRIMORDIAL BLACK HOLES FORMATION}

If density perturbations acquire sufficiently large values, they can cause collapsing regions to produce early structure, causal isolated regions, and possibly primordial black holes $[19$ 21].

The problem of finding the value of the critical overdensity $\delta_{c}$ in perturbations such that a primordial black hole can be formed in the early Universe has been discussed by several authors both analytically and numerically 22 26]. The progress made in this regard is vague and sometimes contradictory. For example, in 1975 Carr [21] obtained that $\delta_{c} \sim w$, where $w$ is the equation of state, while in [22, 23] Niemeyer \& Shibata obtained that the critical value is $\delta_{c}>0.7$ and in [26] Green conclude that a smaller value, $\delta_{c} \sim 0.3$, is a better estimation, this last coincides with the Carr's results for the case of a Universe in radiation domination epoch.

On the other hand, to estimate the mass of a primordial black holes is necessary to calculate the black hole mass function $\beta$ defined as

$$
\beta(M)=\frac{\rho_{P B H}}{\rho_{T o t}}=\int_{\delta_{c}}^{\infty} P(\delta) d \delta,
$$

which measures the contribution of PBH to the total energy density of the Universe. This parameter is constrained by observational evidence over a wide range of masses; the stronger constraint lies on PBHs of mass $M \sim 10^{15} \mathrm{~g}$, whose evaporation time is $\tau \sim$ age of the Universe, so $\beta \leq 10^{-20}$, a value that basically comes from the non-observation of $\gamma$-rays originating on Hawking radiation [19, 27].

Following typical calculations, if the over-dense regions are spherically symmetric with a Gaussian distribution given by

$$
P(\delta)=\frac{1}{\sqrt{2 \pi} \sigma} \exp \left(-\frac{\delta^{2}}{2 \sigma^{2}}\right),
$$

where $\sigma$ is the mass variance at horizon crossing, it is possible to transfer the observational constraint on PBHs from the mass function $\beta$ to the mass variance $\sigma$. Thus, we get a threshold mass variance of $\sigma_{*}=0.08$, any mass variance above this value indicates an over-production of $\mathrm{PBHs}$ which would put a tension between the inflation-preheating model and observations. If instead of a Gaussian distribution (see Eq. (99) a first order chi-squared distribution for density fluctuations is considered, the threshold changes to $\sigma_{*}=0.03$ [7].

Given the curvature perturbation (5), it is possible to define the mass power spectrum by

$$
\mathcal{P}_{\zeta} \equiv \frac{k^{3}}{2 \pi^{2}}\left|\zeta_{k}\right|^{2},
$$

and the mass variance $\sigma$, see Eq. (9), as

$$
\sigma^{2}=\left(\frac{4}{9}\right) \int_{0}^{\infty}\left(\frac{k}{a H}\right)^{4} \mathcal{P}_{\zeta} \tilde{W}(k R) \frac{d k}{k},
$$



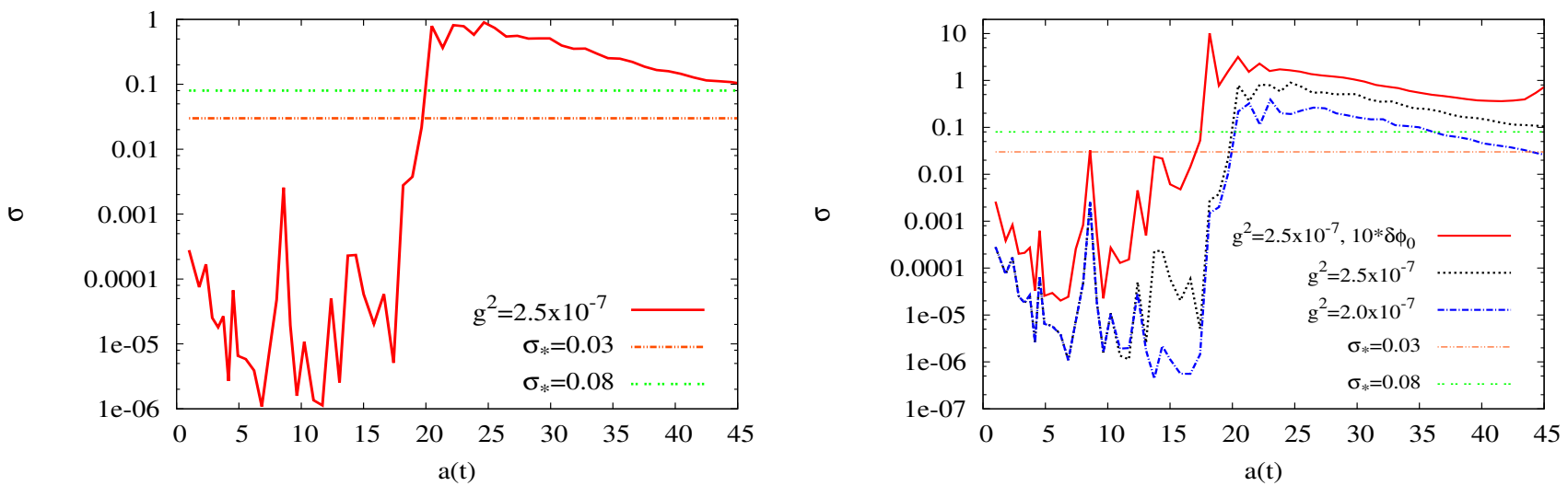

FIG. 2: (Left) The mass variance $\sigma$ grows explosively during the preheating process even exceeding both, $\sigma_{*}=0.03$ and $\sigma_{*}=0.08$ values. (Right) The dependence on coupling constant parameter and on initial amplitude inflaton perturbations. The smoothing scale is $R=1 / a H$. This suggests that this chaotic inflationary model presents PBHs production, although the exact amount depends on the chosen values of the parameters. See the text for more details.

where $\tilde{W}(k R)$ is the window function in Fourier space given by

$$
\tilde{W}(k R) \equiv \exp \left(-k^{2} R^{2} / 2\right),
$$

and $R$ is the artificial smoothing scale 28 ].

The growth of the modes of the energy overdensity, caused by the parametric resonances of $\delta \chi_{k}$ [29], produces the growth of the modes of $\delta \rho_{k}$ and $h_{i j}$. Hence, we expect a growth of the modes of $\zeta_{k}$, so that the spectrum (10) must also grow during the preheating process. These changes should be reflected in the mass variance, which would give us information on how much lumpy the Universe becomes. This phenomenon was observed already in [9] for a quartic inflationary model.

Fig. 2 (left) shows the growth of $\sigma$ during the numerical evolution of the preheating model in (1). It is to be noted that the variance grows explosively during the preheating process, even exceeds the aforementioned bounds $\sigma_{*}=0.03$, and $\sigma_{*}=0.08$, if the smoothing scale is $R \equiv 1 / k_{*}=1 / a H$, see Eq. (12). This is an evidence that overproduction of $\mathrm{PBHs}$ is possible in this model of chaotic inflation for the values of parameters that we have chosen.

The dependence of $\sigma$ on parameters of this model is shown in Fig. 2 (right). It is observed that the amplitude of $\sigma$ strongly depends on both the value of the coupling constant $g$ and of the initial field perturbations, even when the initial amplitudes in the initial fluctuations $10\left(\delta \phi_{0}\right)$ is not a well motivated selection. ${ }^{2}$ In this way, the requirement for the non overproduction of PBHs can be used to add constraints on the values of the model parameters.

In Fig. 2 we also observe that in all cases $\sigma$ decays immediately after the end of preheating, which could give us indications that, in this model, during the beginning of thermalization process, part of the structure has been diluted. On the other hand, Fig. 3 shows the dependence of $\sigma$ on the size of the artificial smoothing scale $R$. If we change the size of the smoothing scale we find that:

1. The maximum variance is reduced significantly even preventing the overproduction of PBHs.

2. The mass variance does not decrease for $R=1 /(0.01 a H)$ at the end preheating.

All of this likely indicates that we need to calculate a scale $R_{*}$ containing only structures that are able of survive the thermalization process. Only these stable structures would be present at the beginning of radiation domination as very early structures.

\section{CONCLUSIONS}

In this work we have solved the system of Einstein equations for a universe at the time of preheating in a chaotic inflationary model. The dynamics of scalar perturbations of both matter and metrics have been evaluated. Using the spectrum of the gauge invariant variable $\zeta$ has been possible to calculate the mass variance $\sigma$.

\footnotetext{
${ }^{2}$ In this case $10\left(\delta \phi_{0}\right)$ means that the initial fluctuations of the fields were tenfold increased from the Bunch-Davies vacuum.
} 


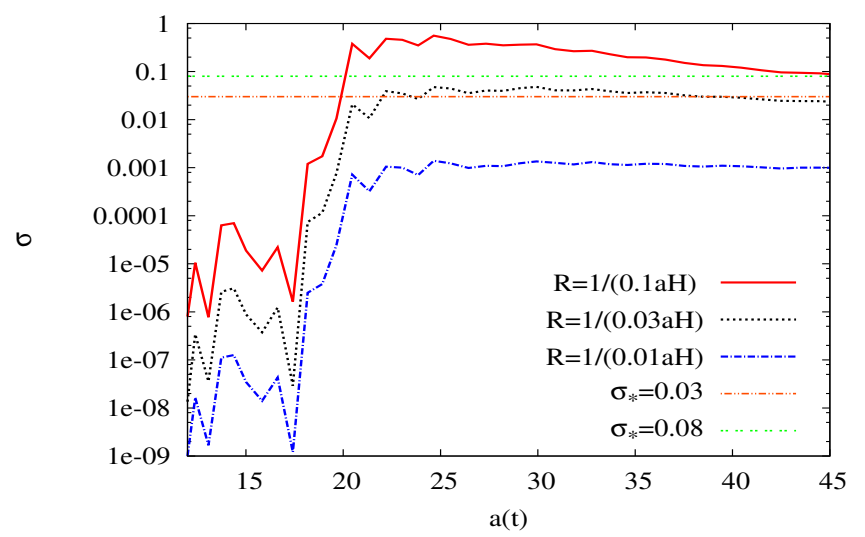

FIG. 3: Time evolution of $\sigma$ for three sizes of the smoothing scale $R$ are shown. The $R=1 /(0.1 a H)$ case shows overproduction but for $R=1 /(0.03 a H)$ the overproduction is reduced. For a artificial smoothing scale $R=1 /(0.01 a H)$ only stable structures are produced, see also the text for more details.

Considering the model (1) with $m=10^{-6} M_{P l}$ and $g^{2}=2.5 \times 10^{-7}$, it was found that the mass variance $\sigma$ exceeds the $\sigma_{*}$ value. We have considered the constraint $\sigma \leq \sigma_{*}=0.08$ which comes from the condition imposed upon the mass fraction $\beta \equiv \rho_{P B H} / \rho_{T O T}$, which is in turn obtained from the observation of the background in gamma rays. The result $\sigma>\sigma_{*}$ could put stress between this inflation-preheating model and observations.

It has been found that the production during preheating can be a generic result even though the results depends on the parameters of model, initial conditions and on the smoothing scale selection. Because the $\delta_{c}$ value has not been determined in an preheating ambiance and then $\sigma_{*}$ value is not well established, the PBHs over-production should be taken with some caution.

We have shown that after the preheating process the structures produced during preheating can be able to survive the thermalization process. This structures possibly would be present at the beginning of Hot Big-Bang era.

More research is needed in this area, and we are working on the production of PBH in a more general inflationary model. The results will be reported elsewhere.

[1] A. L. Erickcek and K. Sigurdson, Phys.Rev. D84, 083503 (2011), 1106.0536.

[2] F. Finelli and R. H. Brandenberger, Phys.Rev.Lett. 82, 1362 (1999), hep-ph/9809490.

[3] K. Jedamzik, M. Lemoine, and J. Martin, JCAP 1004, 021 (2010), 1002.3278.

[4] J. Hidalgo, L. A. Urena-Lopez, and A. R. Liddle, Phys.Rev. D85, 044055 (2012), 1107.5669.

[5] B. A. Bassett, D. I. Kaiser, and R. Maartens, Phys.Lett. B455, 84 (1999), hep-ph/9808404.

[6] B. A. Bassett, F. Tamburini, D. I. Kaiser, and R. Maartens, Nucl.Phys. B561, 188 (1999), hep-ph/9901319.

[7] A. M. Green and K. A. Malik, Phys.Rev. D64, 021301 (2001), hep-ph/0008113.

[8] M. Khlopov, B. Malomed, and I. Zeldovich, Mon.Not.Roy.Astron.Soc. 215, 575 (1985).

[9] B. A. Bassett and S. Tsujikawa, Phys.Rev. D63, 123503 (2001), hep-ph/0008328.

[10] T. Suyama, T. Tanaka, B. Bassett, and H. Kudoh, JCAP 0604, 001 (2006), hep-ph/0601108.

[11] Z. Huang, Phys.Rev. D83, 123509 (2011), 1102.0227.

[12] C. Bennett, D. Larson, J. Weiland, N. Jarosik, G. Hinshaw, et al. (2012), 1212.5225.

[13] C.-P. Ma and E. Bertschinger, Astrophys.J. 455, 7 (1995), astro-ph/9506072.

[14] J. M. Bardeen, P. J. Steinhardt, and M. S. Turner, Phys. Rev. D 28, 679 (1983), URL http://link.aps.org/doi/10.1103/PhysRevD.28.679

[15] D. Wands, K. A. Malik, D. H. Lyth, and A. R. Liddle, Phys. Rev. D 62, 043527 (2000), URL http://link.aps.org/doi/10.1103/PhysRevD.62.043527

[16] T. S. Bunch and P. C. W. Davies, Proceedings of the Royal Society of London. A. Mathematical and Physical Sciences 360, 117 (1978), http://rspa.royalsocietypublishing.org/content/360/1700/117.full.pdf+html, URL http://rspa.royalsocietypublishing.org/content/360/1700/117.abstract

[17] J. F. Dufaux, G. N. Felder, L. Kofman, M. Peloso, and D. Podolsky, JCAP 0607, 006 (2006), hep-ph/0602144.

[18] D. I. Podolsky, G. N. Felder, L. Kofman, and M. Peloso, Phys.Rev. D73, 023501 (2006), hep-ph/0507096.

[19] S. Hawking, Mon.Not.Roy.Astron.Soc. 152, 75 (1971).

[20] I. D. Zel'dovich, Ya. B.and Novikov, Sov. Astron. A. J. 10, 602 (1967).

[21] B. J. Carr, Astrophys.J. 201, 1 (1975). 
[22] J. C. Niemeyer and K. Jedamzik, Phys.Rev. D59, 124013 (1999), astro-ph/9901292.

[23] M. Shibata and M. Sasaki, Phys.Rev. D60, 084002 (1999), gr-qc/9905064. http://link.aps.org/doi/10.1103/PhysRevLett.80.5481

[25] I. Musco, J. C. Miller, and L. Rezzolla, Class.Quant.Grav. 22, 1405 (2005), gr-qc/0412063.

[26] A. M. Green, A. R. Liddle, K. A. Malik, and M. Sasaki, Phys.Rev. D70, 041502 (2004), astro-ph/0403181.

[27] B. J. Carr (2005), astro-ph/0511743.

[28] A. R. Liddle and D. H. Lyth, Phys.Rept. 231, 1 (1993), astro-ph/9303019.

[29] L. Kofman, A. D. Linde, and A. A. Starobinsky, Phys.Rev. D56, 3258 (1997), hep-ph/9704452. 